\title{
THICK FILM FAIL-SAFE RESISTORS
}

\author{
ST. NOWAK and D.L. WOJCICKA \\ Research and Development Centre of Hybrid Microelectronics and Resistors Unitra-Telpod, Cracow, \\ Poland
}

(Received June 5, 1981; in final form June 26, 1982)

The investigations of low resistance thick film fail-safe resistors are presented. Particularly the shape of a resistive path; the temperature of the central part of the resistor while increasing the power up to 4 Watts; the kind of composition material for the resistive path vs. extensometer effect, and the stability of the resistor are discussed.

\section{INTRODUCTION}

With the growth of the complexity of electronic circuits, the necessity of their protection seems to be indispensable. In particular, high power circuits should be safety protected. The fail-safe problem is of considerable width and of great significance for automatic control engineering. Damage of an electronic element can bring about serious consequences, as e.g. fire, hazards dangerous to human life, destruction of expensive devices or their elements, destruction of a batch during the technological process, etc.

Constructors of electronic circuits, including complex ones, design them in such a way as to avoid these grave and negative effects in the case of damage. One of the measures is to apply fail-safe resistors in suitably chosen or specially designed branches of an electronic circuit. ${ }^{1}$

Fail-safe resistors known to date are wire-wound resistors ${ }^{2}$ made, for example, by Micron Electric Co. Ltd. ${ }^{3}$ The production of these resistors is labout consuming when high resistance is involved due to a very thin resistive wire, and at very low resistance values it is difficult to obtain a hot spot because of using a thick wire.

\section{EXPERIMENTAL RESULTS AND DISCUSSION}

For years our Research Centre has been interested not only in hybrid circuits but also in thick film resistors of up to 16 Watts power. Based on the experiments made, a thick film fail-safe resistor has been designed. ${ }^{4,5}$ At normal operation the power of 1 Watt is dissipated in this resistor. However, when damage occurs in the electric circuits causing the dissipation of the power of more than 4 Watts in the fail-safe resistor, a break is safety formed in it.

\subsection{Resistor Properties}

- Nominal power 1 Watt

- Resistance range $0.1 \Omega$ to $1 \mathrm{k} \Omega$

- Resistance tolerance $\pm 5 \%$ and $\pm 10 \%$

- Changes in resistance after 1000 hours at $P_{n}$ smaller than $5 \%$ at an ambient temperature of $+70^{\circ} \mathrm{C}$ 
- The size of the substrate of $96 \% \mathrm{Al}_{2} \mathrm{O}_{3}$ equals $5 \times 10 \times 0.5 \mathrm{~mm}$

- The resistor body is encapsulated in a thermocontractible tube

- The shape of the resistor is presented in Figure 1

\subsection{Solutions of Problems}

Two essential problems were solved during the preparation of this resistor, viz.

- resistance stability during application at the power of 1 Watt at an ambient temperature of $+70^{\circ} \mathrm{C}$

- effectiveness of 'damage in a safe way' at the power of more than 3 Watts during ca 5 minutes.

In order to attain such effects, it was necessary to analyze and study a few questions which concerned:

- approximate calculation of the maximum temperature of the resistor,

- determination of the hot spot area and the shape of isotherms by means of thermovision through the change of the resistive path shape,

- determination of the temperature diagram along the resistor main axis by means of thermovision,

- testing of the extensometer effect of resistors made of various compositions,

- stability tests at the time of 1000 hours

- analysis of the investigation results and conclusions.

Two assumptions were accepted for the thermal analysis of the resistor, i.e.

a) the increase in temperature is proportional to the loss of power.

The following straight line equation was obtained from calculation

$$
\Delta \mathrm{T}_{1}=2.57 \cdot 29.9 \mathrm{P}
$$

where the coefficient 2.57 results from the shape of the substrate and the shape of the resistor, whereas the coefficient 29.9 is from the substrate material and its thickness and $P$ denotes electric power.

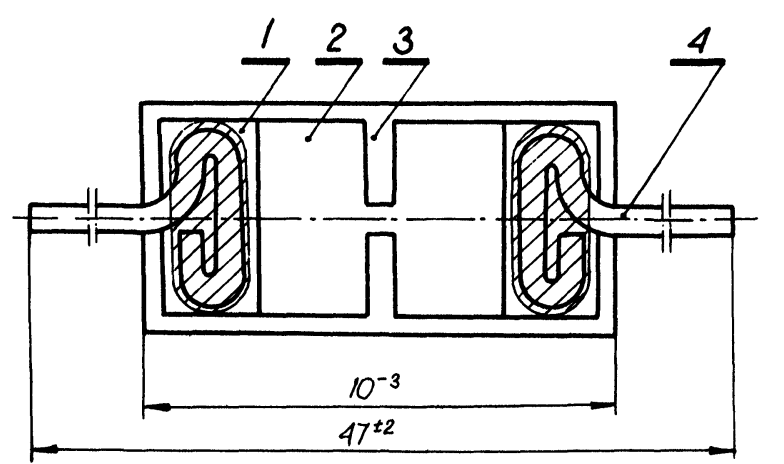

1) electrode layer

2) resistive layer

3) narrowing area of the resistive path

4) wire lead

FIGURE 1 Fail-safe resistor without encapsulation. 
b) the increase in temperature is connected with the power lost in the resistor by the exponential function. The following equation was obtained from calculation and measurements:

$$
\Delta \mathrm{T}_{2}=29.9 \exp / 0.5 \mathrm{P} /
$$

The coefficient 29.9 is the material coefficient as in (a). The coefficient of shape is connected with the index of the power 0.5 . This value can be chosen from the measurements.

Figure 2 presents both curves and the dependences 1 and 2 shown by thermovision for two resistors.

Both $\Delta \mathrm{T}_{1}$ and $\Delta \mathrm{T}_{2}$ are not strictly accurate approximations. With the straight line $\Delta \mathrm{T}_{1}$ errors are smaller. The curve $\Delta \mathrm{T}_{2}$ has a form approximating empirical curves and is nearly parallel to curve 1 .

It should be noted that exponential approximation does not solve the problems of low power, because the increase of temperature for $\mathrm{P}=0$ ought to equal zero. This does not happen with the curve $\Delta \mathrm{T}_{2}$.

The above divergence being taken into account, our efforts were concentrated on examining the distribution of isotherms at various constructional solutions of resistive paths. Isotherms were determined by photographing the thermovision images. ${ }^{6}$ Figure 3 presents two from among numerous solutions, i.e. 3.1 a and 3.2 a, which allow one to draw conclusions concerning the choice of the resistive path design of the fail-safe resistor.

Isotherms copied from colour slides are shown in Figures $3.1 \mathrm{~b}$ and $3.2 \mathrm{~b}$.

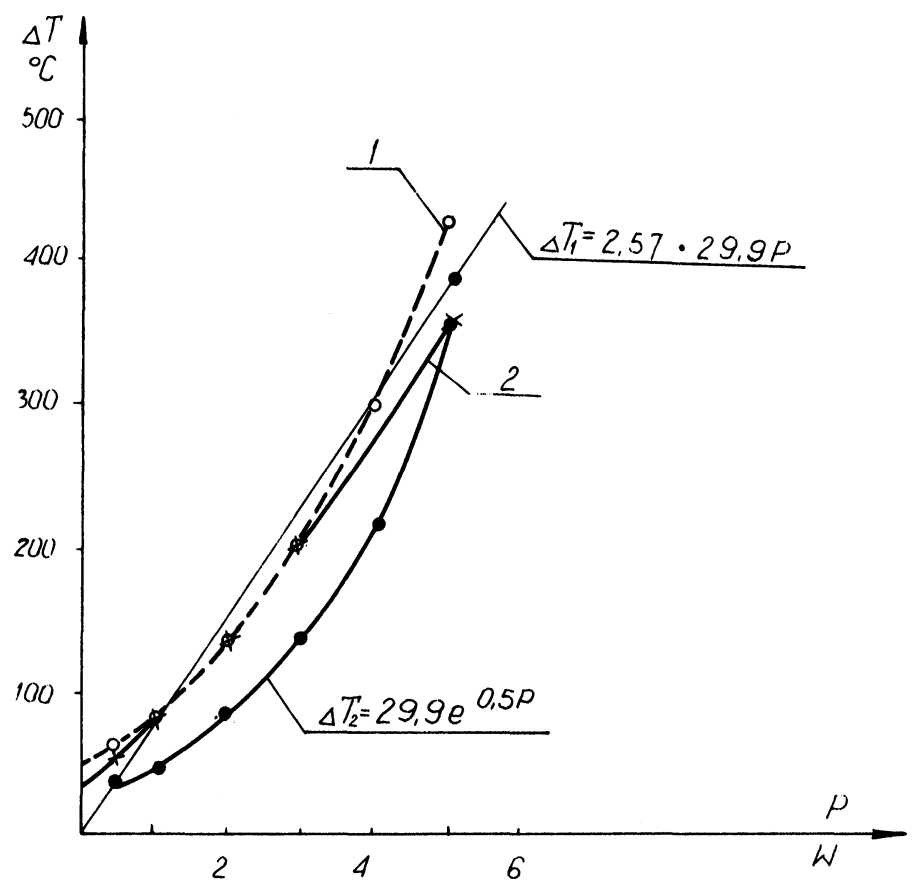

1 and 2 curves for two resistors obtained by means of thermovision. Straight line $\Delta T_{1}$ and curve $\Delta T_{2}$ obtained from calculation.

FIGURE 2 Dependence of the temperature increase $\Delta T$ at the hottest point on power $P$ lost in the resistor. 


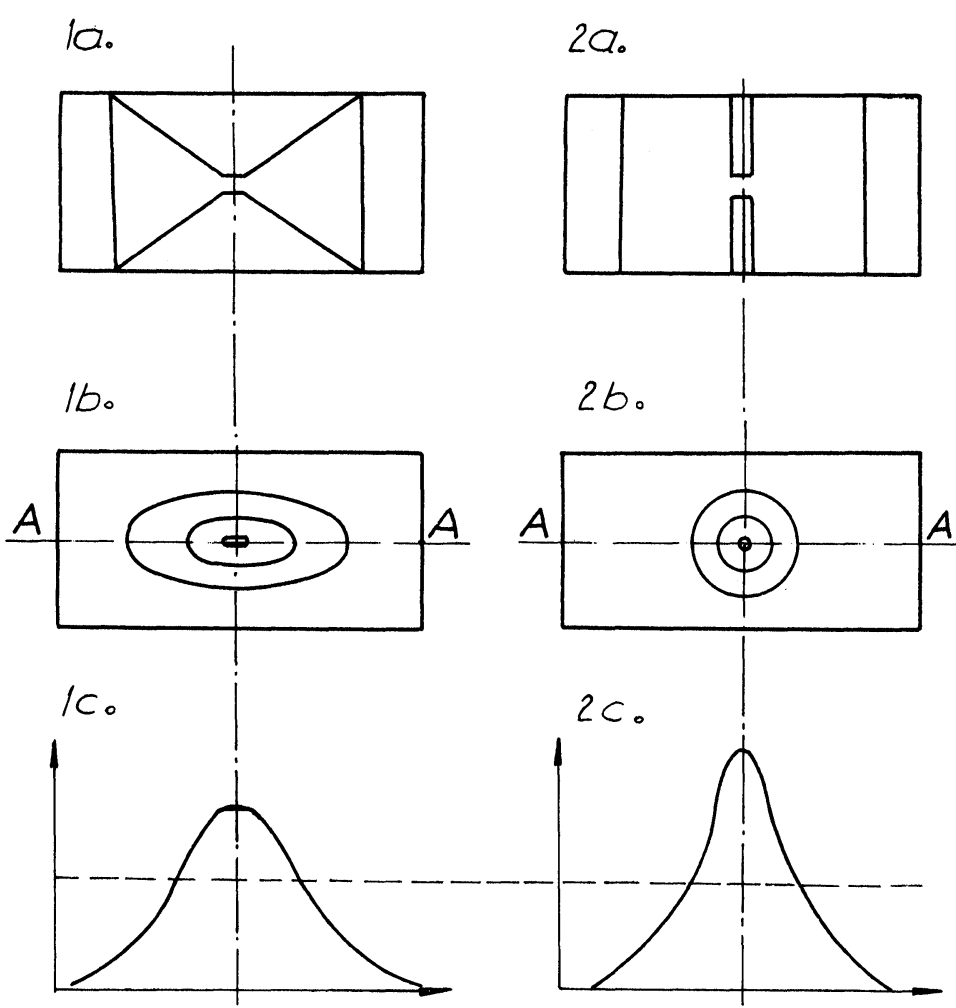

FIGURE 3 Effect of the resistive path shape on the isotherm shape and on hot spot maximum temperature.

Figure $3.1 \mathrm{c}$ and Figure $3.2 \mathrm{c}$ present diagrams obtained through thermovision along the resistor main axis. The axis is denoted by lineA-A in Figures $3.1 \mathrm{~b}$ and $3.2 \mathrm{~b}$.

The more effective protection-ensured by the resistor, the slenderer is the curve in Figure $3.2 \mathrm{c}$, and due to this larger $\Delta \mathrm{T}$ occurs at the same power in the resistor. However, it should be remembered that the growth of the slenderness of this curve is followed by a rise in temperature at maximum power. Therefore, after having chosen the conditions affecting the protection effect, the stability test should be made employing nominal power and a maximum ambient temperature.

This general analysis and study of the fail-safe problem being completed, the construction of a series of resistors was started. When an alloy conductive compositon was used for a resistive path very good parameters were obtained. When a resistive paste was applied, difficulties in ensuring its proper stability was encountered. The main cause of instability was the change due to the extensometer effect, the results of which investigation have been presented in Figure 4.

Figure $4 \mathrm{a}$ concerns the resistor of $3 \mathrm{ohms}$ made of a resistive paste and shows mean changes of $\bar{\delta}$ and an interval $\pm 3 \sigma$ in the function of pressure onto the resistor substrate. Maximum values reach $+10 \%$ at loading of $3 \mathrm{kG}$, and ca $+5 \%$ at a loading of $1.5 \mathrm{kG}$. Figure $4 \mathrm{~b}$ illustrates the interval $\pm 3 \sigma$ for the resistor of $3 \mathrm{ohms}$ made of a conductive alloy paste. The changes presented in this figure can be neglected, since the mean value does not exceed $\pm 0,1 \%$, and the maximum values are within the limits of $0,4 \%$. Both tests were made under identical conditions similar to the conditions and stresses which 


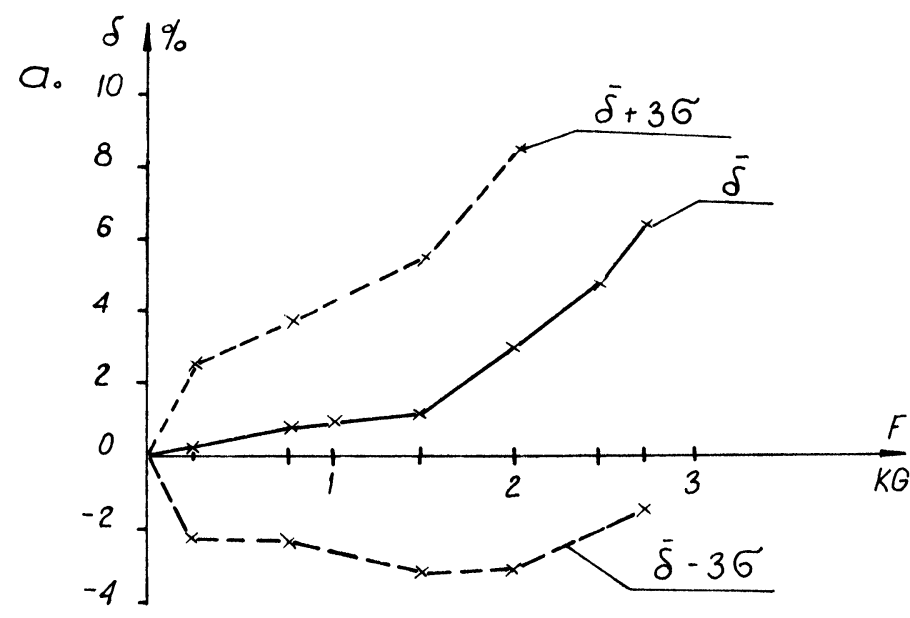

resistors of $3 \mathrm{ohms}$ of a resistive paste resistors of $3 \mathrm{ohms}$ of an alloy conductive paste

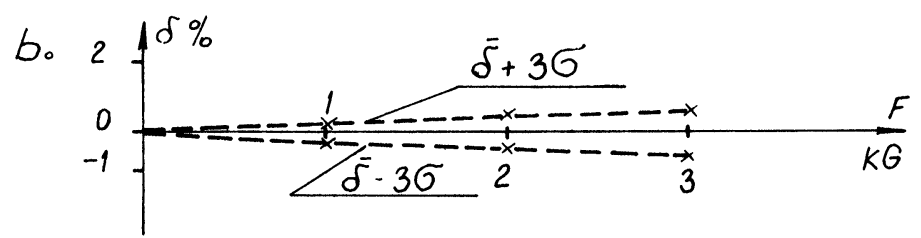

FIGURE 4 Resistance changes in the bending force function.

occur during the work of the assembled resistor; pressure being applied from the side opposite to the resistive film. In these two cases the pressure from the side of the resistive film gave smaller resistance changes.

Simultaneously the stability test was made during 1000 hours at an ambient temperature of $+70^{\circ} \mathrm{C}$ and at power loading of 1 Watt.

In Figure 5 the curves denoted by 1 represent the mean values $\bar{\delta}$, whereas the curves denoted by 2 show the maximum changes $\delta \max =\bar{\delta}+3 \sigma$ of the fail-safe resistors.

It can be seen from the comparison of the curves that the stability of the resistors made of a conductive alloy paste is much better than that of the resistors made of a resistive paste. With the curves for the resistors of $3 \mathrm{ohms}$ made of a conductive composition the maximum changes of $\delta$ max do not exceed $1.5 \%$, and the mean ones are close to zero. With resistive compositions the mean value reaches $2,5 \%$ and the maximum one equals ca. $5 \%$, i.e. it is on the verge of the requirements this type resistors have to meet.

Despite the low resistivity of ca. $0.35 \Omega / \square$ of conductive alloy compositions and some technological disadvantages encountered when resistors of relatively high resistance i.e. 3 ohms are produced from these pastes, their stability in the life test, as well as the insignificant extensometer effect were the factors deciding the choice of this composition for the production of fail-safe resistors.

\section{CONCLUSION}

1) A thick film resistor can operate as a fail-safe resistor.

2) The smallest hot spot area has been obtained with the resistive path design according to Figure 1. ie narrowing of the resistive path equalling $0.6 \times 0.6 \mathrm{~mm}$. 


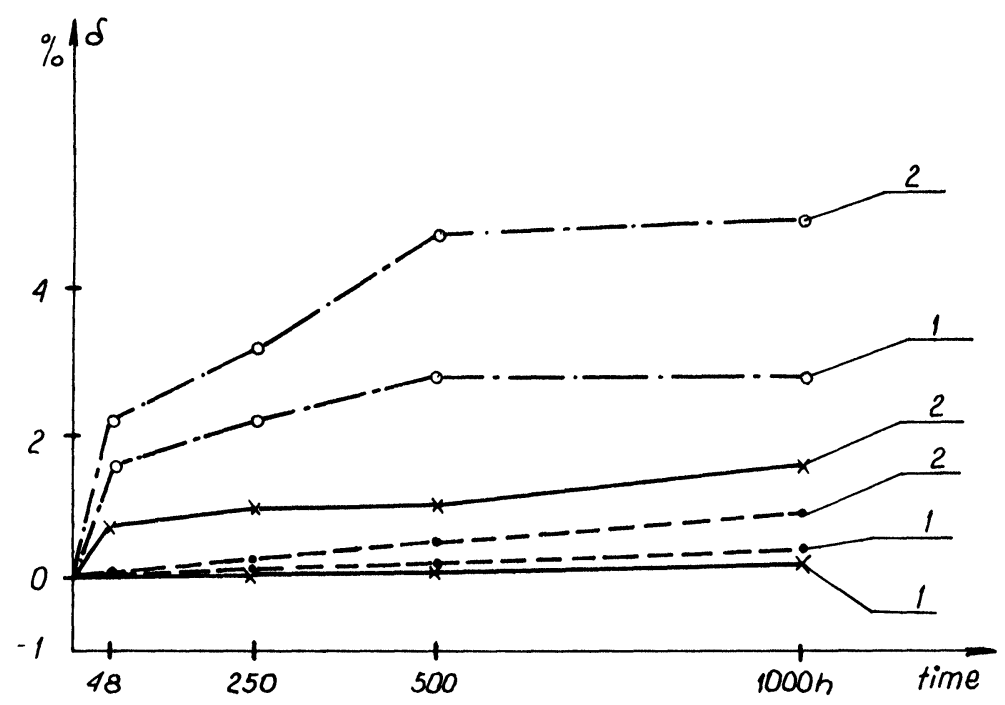

FIGURE 5 Resistance changes $\delta$ in $\% \%$ during stability test - - $--0.68 \mathrm{ohm}-$ conductive alloy paste, $3 \mathrm{ohms}-$ conductive alloy paste, -. - - - $-3 \mathrm{ohms}$ - resistive paste.

3) Resistive compositions containing ruthenium compounds of the resistivity ca. $1 \Omega / \square$ do not produce sufficiently stable resistors mainly owing to a considerable extensometer effect.

4) To produce low resistance fail-safe resistors, conductive alloy compositions of 0.1 to $0.4 \Omega / \square$ were employed; stability and load carrying ability of these resistors were good.

5) The results of the approximate theoretical calculations of temperature depart from those obtained in practice.

6) Basic conclusions concerning the resistor construction were obtained from contactless photographs of the temperature distribution on the resistor by means of thermovision, at power loadings of 0.5 Watt to $4.0 \mathrm{Watt}$ at the time up to $10 \mathrm{sec}$.

7) Isotherms on the fail-safe resistor should have a ring shape or that of an ellipse whose longer diameter ought to be perpendicular to the resistors main axis. On the other hand, the effect shown in Figure $3.2 \mathrm{c}$ signals the deterioration of the protective ability.

8) At power loadings of 3 to 4 Watts during 5 min. every resistor subjected to testing was 'safely' damaged.

\section{REFERENCES}

1. T. Hasegawa "Graceful degradation in road traffic control system", II USA-Japan Computer Conference (1975).

2. Patent USA 3.792.406, (1974).

3. Catalogue dates "Micron cermet resistors" Research Report of Welwyn Electric Limited, (1972).

4. D.L. Wojcicka "Fusible cermet resistors" Research Report of Welwyn Electric Limited, (1972).

5. Complete Polish Patent Specification, P-206211, (1978).

6. R.F. Szeloch, J. Kozlowski "Thermovision measurement of thick film resistors", Report of Technical University of Wroclaw, (1980). 

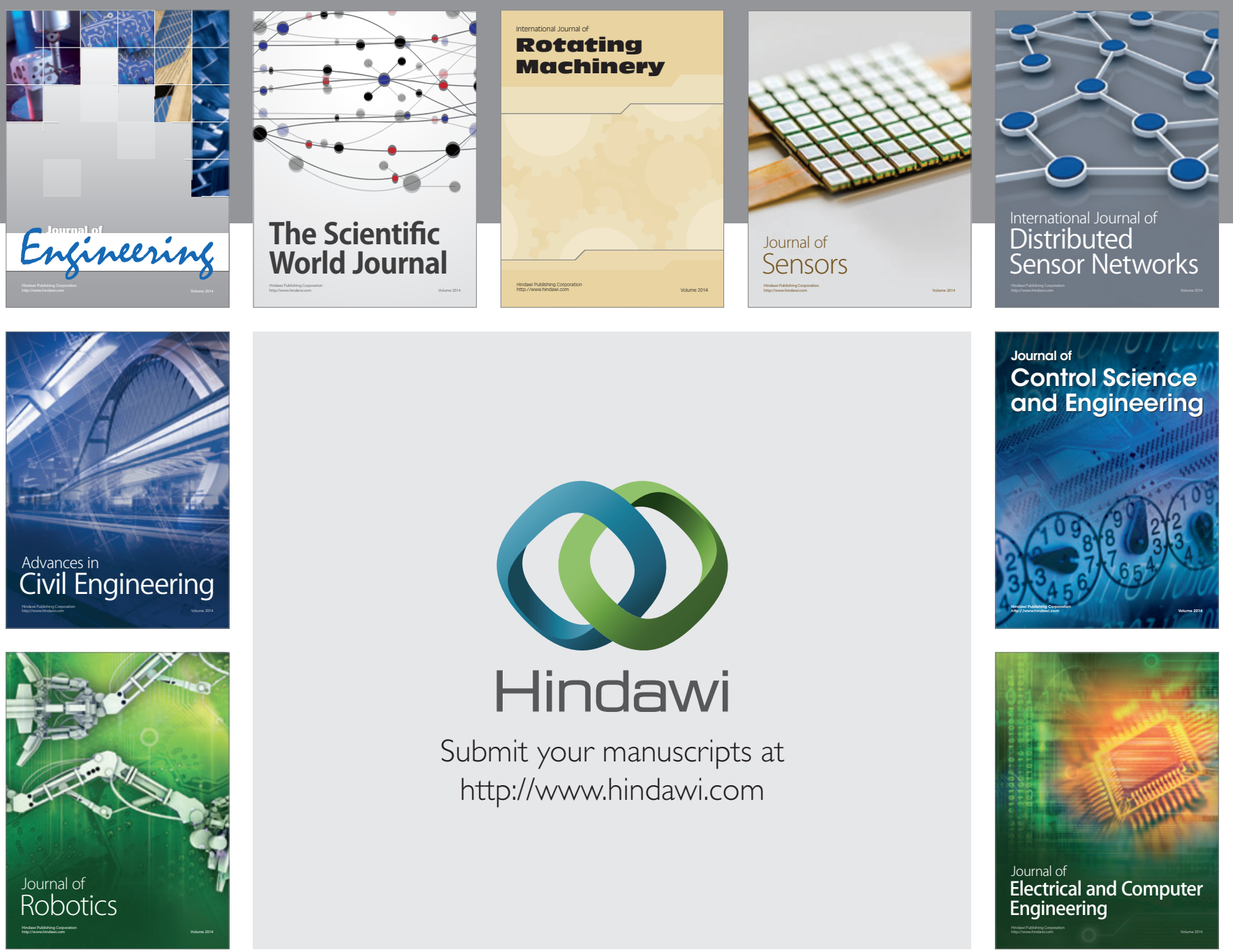

Submit your manuscripts at

http://www.hindawi.com
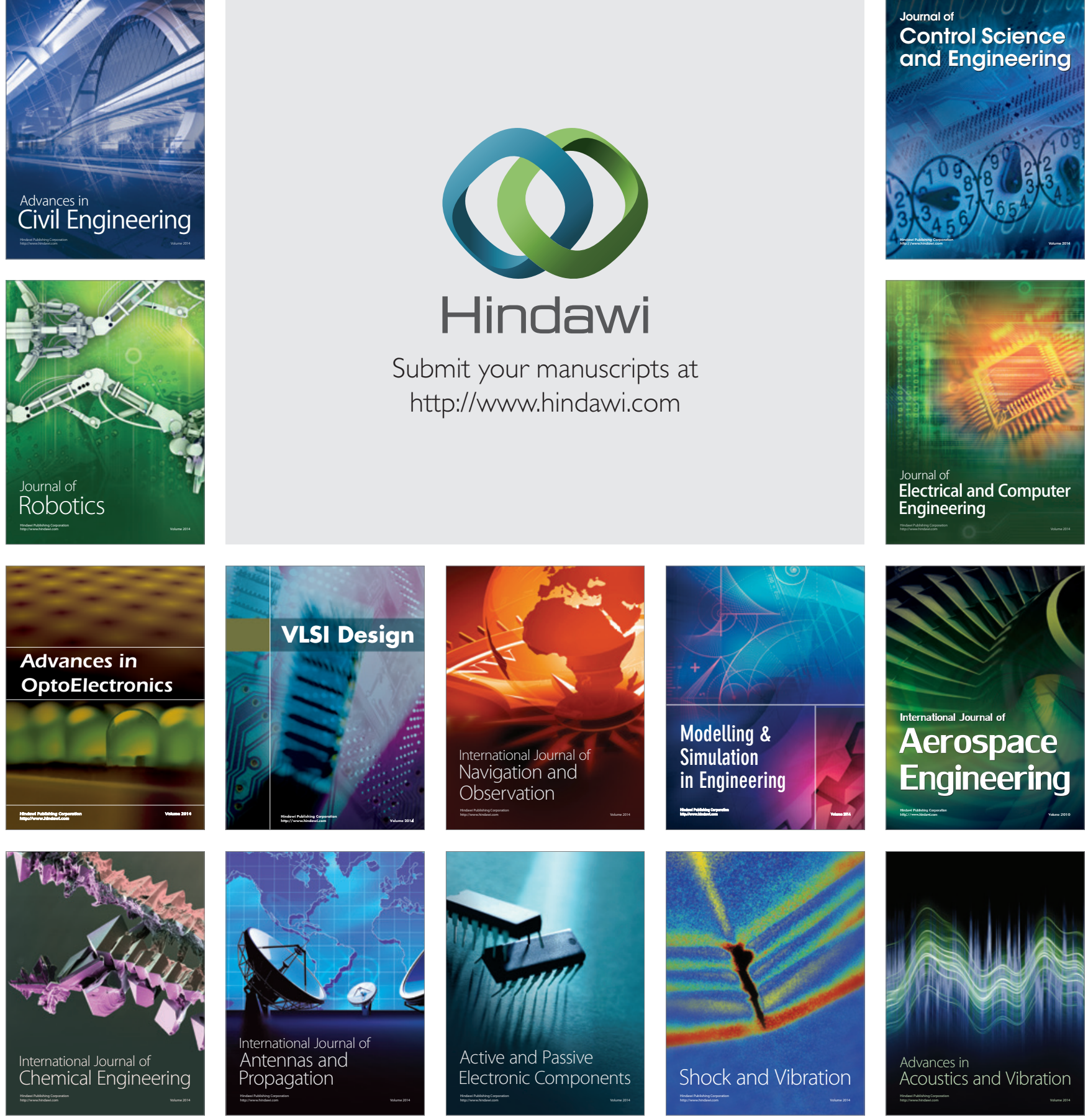